\title{
Notch1 Signaling Regulates Wound Healing via Changing the Characteristics of Epidermal Stem Cells
}

\author{
Bin Shu ${ }^{1}$, Rong-Hua Yang ${ }^{2}$, Yan Shi ${ }^{3}$, Ying-Bin Xu${ }^{1}$, Jian Liu ${ }^{1}$, Peng Wang ${ }^{1}$, Xu-Sheng Liu ${ }^{1}$, Shao-Hai Qi ${ }^{1}$ and Ju-Lin Xie ${ }^{1 *}$
}

${ }^{1}$ Department of Burns, First Affiliated Hospital of Sun Yat-sen University, Guangzhou, Guangdong 510080, China

${ }^{2}$ Department of Burns, Foshan First People's Hospital, Foshan, Guangdong 528000, China

${ }^{3}$ Department of Plastic, Jiangxi Women and Children Hospital, Nanchang, Jiangxi 330006, China

\begin{abstract}
During wound healing and reconstruction, epidermal stem cells (ESCs) migrate to the wound site and activate to repair the damaged epithelium. Moreover, there exist complicate signaling pathways to regulate wound regeneration including Notch signaling. The Notch signaling pathway is a regulator of epidermal differentiation, which may be an important mediator of wound regeneration that participates in various processes, from the development of the dermis to the formation of skin appendages. Here, we show that Notch signaling pathways are upregulated by Jagged1 in ESCs and stem cell characteristics of ESCs change when Notch1 signaling varies. By administration of siRNAJagged1 knockdown ESCs in wounds, we observe that the suppression of Jagged1 down regulate expression of Notch signaling and resulted in poor-quality wound healing. Connecting Notch1 pathways activity to ESCs response to wound repairing may develop a new therapeutic strategy for delayed healing.
\end{abstract}

Keywords: Notch1 signaling; Epidermal stem cells; Wound healing

\section{Introduction}

The skin is the largest organ in the human body and functions as a barrier from environmental aggressions, such as external microorganisms or dehydration. Trauma to the skin can arise from abrasions, lacerations, and thermal, electrical, or chemical burns [1]. As a response to injury, several overlapping events of precise sequence occur, including inflammation, granulation tissue formation, reepithelialization, and matrix formation and remodeling. The success of wound healing process depends on growth factors, cytokines, and chemokines involved in a complex integration of signals that coordinate cellular processes [2]. Various factors contribute significantly to this precise sequence, which results in either delayed wound healing or excessive healing (as in hypertrophic and keloid scars). Given the complexity of the wound repair process, exploring the mechanisms of wound healing is useful for the improvement of wound healing and the inhibition of scar formation.

With the ongoing advances in biological research, the effects and function of epidermal stem cells (ESCs) have gained increasing attention and have become a subject of concern. ESCs can switch rapidly and reversibly between quiescence and activity following injury and/or drug treatment [3]. The self-renewal and wound repair abilities of ESCs mainly depend on their compensatory proliferation and oriented differentiation [4]. Wound healing is not only relevant to the amount of ESCs but also to their differentiation. Activated ESCs migrate to the wound site for the replenishment of lost cells to aid in the re-epithelialization and repair of the damaged epithelium [5]. ESCs have also been shown to stimulate dermal collagen synthesis and enhance the tensile strength recovery of skin [6]. Under the correct guidance of signals, ESC differentiation contributes to wound repair. Conversely, when incorrect signals are received, the differentiation changes and abnormal wound repair occurs [7]. Thus, the mechanism of signaling pathways related to ESC proliferation and differentiation should be explored because these actions may improve the speed and quality of wound healing and promote the structure and function of physiological repair. ESC proliferation and differentiation are largely regulated by the microenvironment or the stem cell "niche", which comprises the cell components in the microenvironment of stem cells and the signaling pathways supporting the stem cells, has a key function in the regulation of migration, proliferation, and differentiation of stem cells through a network of multiple overlapping signaling pathways [8].

Notch pathway has a key linking the control of epidermal homeostasis and differentiation. Notchl signaling is active in the suprabasal cells of the epidermis. Notch signaling was initially reported to be required for cell differentiation in the epidermis, with the loss of Notch in the epidermis resulting in the hyperplasia of epidermal cells and the loss of expression of differentiation markers [9]. There is increasing evidence to suggest that aberrant Notch1 signaling may contribute directly to delayed wound healing and altered expression of Notch receptors identified in pathological scar [10,11]. However, the role of Notch signaling in wound healing has not been as extensively studied as have some other pathways, and there are still many questions to explore. Recent evidence has suggested that Notch signaling plays a pivotal role in wound repairing, including accelerated wound closure, pro-migratory effects of fibroblast and vascular endothelial cells, increased collagen deposition and vascularity, and that it is required for proper healing [12-14]. In addition, Notch is well known as a regulator of epidermal differentiation of skin $[9,15,16]$, but its role in this process during the re-epithelialization of healing wounds is relatively unexplored.

Here, we discuss the role of Notch1 signaling pathways in cutaneous wound repair, ranging from complete regeneration to scar tissue formation. In addition, we investigate whether Notch1 signaling regulates ESCs proliferation and differentiation. Finally, we observe the effects that siRNA-mediated knockdown of Jagged1 in ESCs on

${ }^{*}$ Corresponding author: Ju-Lin Xie, Department of Burns, First Affiliated Hospital of Sun Yat-sen University, Guangzhou, Guangdong 510080, China, E-mail: xiej190@sina.com

Received June 23, 2016; Accepted July 01, 2016; Published July 07, 2016

Citation: Shu B, Yang RH, Shi Y, Xu YB, Liu J, et al. (2016) Notch1 Signaling Regulates Wound Healing via Changing the Characteristics of Epidermal Stem Cells. J Stem Cell Res Ther 6: 348. doi:10.4172/2157-7633.1000348

Copyright: (c) 2016 Shu B, et al. This is an open-access article distributed under the terms of the Creative Commons Attribution License, which permits unrestricted use, distribution, and reproduction in any medium, provided the original author and source are credited. 
Citation: Shu B, Yang RH, Shi Y, Xu YB, Liu J, et al. (2016) Notch1 Signaling Regulates Wound Healing via Changing the Characteristics of Epidermal Stem Cells. J Stem Cell Res Ther 6: 348. doi:10.4172/2157-7633.1000348

Page 2 of 10

wound healing. Elucidating the molecular mechanisms controlling the behavior of ESCs will provide novel strategies for the treatment of chronic wound.

\section{Results}

Notch1 signaling pathway is activated in response to wounding and there exist some relationship between Notch 1 and ESCs during wound repairing

To observe the expression of Notch1 signaling and relationship with ESCs in the course of cutaneous wound repairing, fullthickness wounds were made on the middle dorsal skin of SpragueDawley rats which were labeled with BrdU. Expression of the Notch receptors in sections of normal and injured rat skin was analyzed by immunohistochemistry and immunofluorescence. We found that Notch1 with low expression was localized to suprabasal cells in unwounded adult rat skin. During wound healing, expression of Notch1 was appeared in the whole epidermis, especially in basal lamina, and obviously higher than that in the unwounded skin which was peaked on $7 \mathrm{~d}$ and remained significantly elevated until 30d (Figure 1A). Studies have demonstrated that ESCs participate in wound repairing. Thus, we tested whether there exist a relationship between Notch1 and ESCs by Hes1 and BrdU double staining. Compared with unwounded skin, double immunofluorescence staining revealed that co-localization of these two proteins in the epidermis was increased on $7 \mathrm{~d}$ post-wounded, indicating that the expression of Notch1 pathway and ESCs were improved during wound repairing (Figure 1B). These results implied that Notch1 signaling pathway was involved in wound repairing which induced by ESCs.

To determine whether Notch1 signaling pathway involved in wound healing, we measured the protein level of the component of Notch1, such as Jagged1, Notch1 and Hes1. The protein level of Jagged1, Notch1 and Hes1 was significantly higher throughout the 30d study period which was peaked on $7 \mathrm{~d}$ in post-wound rat compared to that in unwounded controls (Figure 1C). These data suggest that Notch1 signaling pathway can be activated by wounding which maybe interacted with ESCs during wound repairing.
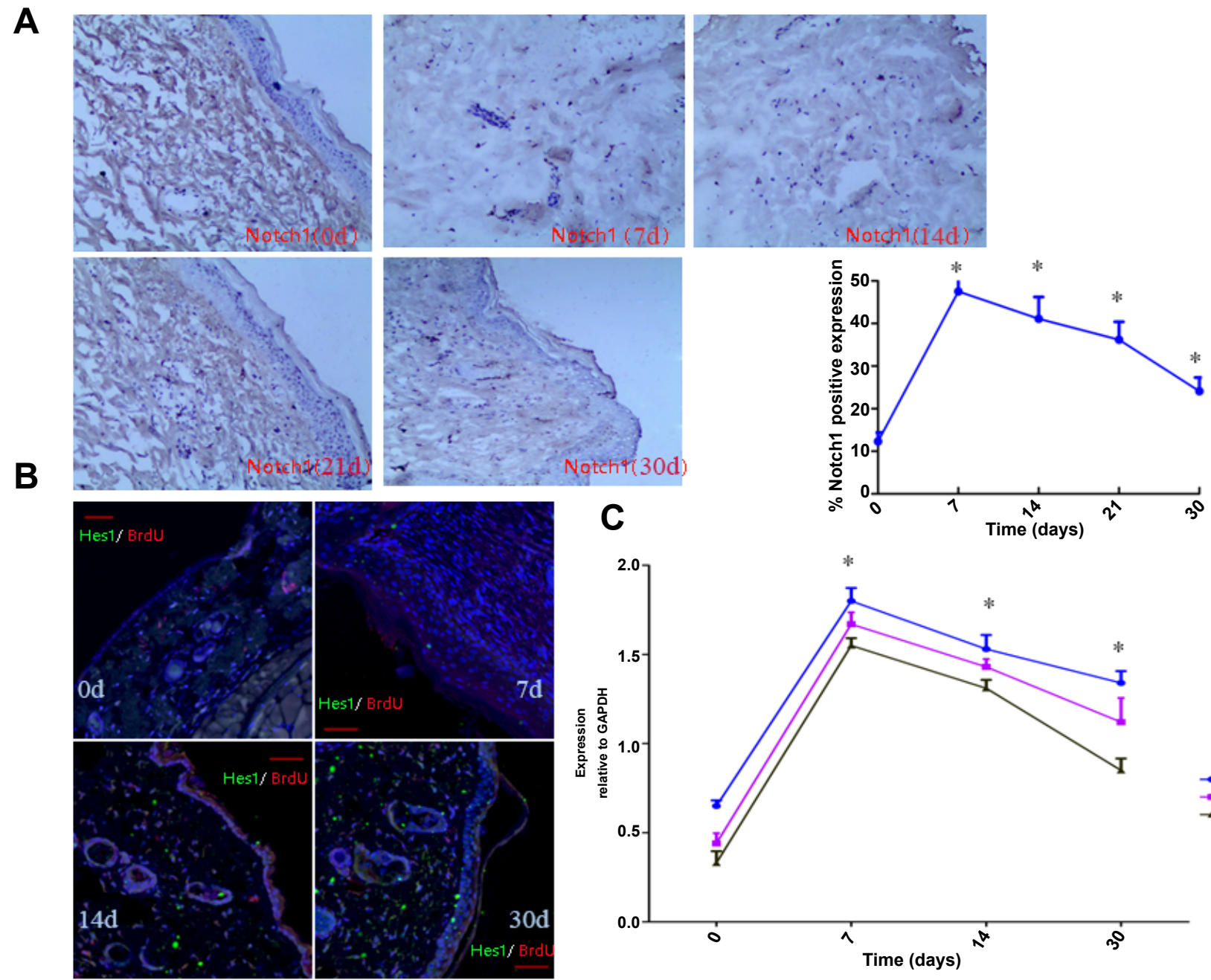

Figure 1: The expression of Notch1 signaling pathway is changed in response to wounding and there are some relationship between Notch1 and ESCs during wound repairing. (A) The expression of Notch1 was appeared in whole epidermis, especially in basal lamina, and obviously higher than that in unwounded skin which was peaked in $7 \mathrm{~d}$ and remained significantly elevated until $30 \mathrm{~d}$. ${ }^{*} P<0.05$ (B) Double immunofluorescence staining with Hes 1 and BrdU revealed that co-localization of these two proteins in epidermis was increased on $7 \mathrm{~d}$ post wounded. (C) The protein level of Jagged1, Notch1and Hes1 were significantly higher throughout the 30d study period which were peaked on $7 \mathrm{~d}$ in post-wound rat compared to that in unwounded controls. ${ }^{*} P<0.05$. 
Citation: Shu B, Yang RH, Shi Y, Xu YB, Liu J, et al. (2016) Notch1 Signaling Regulates Wound Healing via Changing the Characteristics of Epidermal Stem Cells. J Stem Cell Res Ther 6: 348. doi:10.4172/2157-7633.1000348

Page 3 of 10

\section{Wound healing is promoted by Notch 1 activator and impaired by Notch1 inhibitor}

To binding the receptor of Notch1 to affect downstream signaling events, rhNF- $\mathrm{BB}$ and DAPT function as opposite effect regulator of Notch 1 signaling. To test the involvement of rhNF- $\kappa B$ and DAPT in Notch1 signaling in wound healing, we compared the rate of wound healing in rats with enhancing Notch1 expression ( rhNF- $\kappa \mathrm{B}$ group) and inhibiting Notch1 expression by DAPT or control group.

In control rats, $1.0 \mathrm{~cm}$ full-thickness dermal wounds healed rapidly with the lesions being reduced by granulation and re-epithelialization (Figure 2A) and were completely healed within 16 days. We next treated the wounds of normal rats with the secretase inhibitor DAPT to inhibit the activation of Notch1 within cells involved in wound healing. Compared to control group, those treated with DAPT exhibited a significant delay in wound healing. To further confirm the role of
Notch1 signaling in wound healing, we treated the wounds of normal rats with mouse rhNF- $\kappa B$ to activate the Notch1 cells within the wound area. Rats treated with rhNF- $\kappa \mathrm{B}$ showed significantly enhanced wound healing compared to control animals (Figure 2B).

Because Notch1 signaling is varied followed by wounding, we were interesting in whether regulation of Notch1 would affect wound healing. To investigate this possibility, we measured Notch1 activation in response to stimulation with either rhNF- $\kappa \mathrm{B}$ or DAPT. As predicted, rhNF- $\kappa \mathrm{B}$ up-regulated the expression of Notch1 component, whereas DAPT administration inhibited Notch1 signaling pathway. Comparison of the responses to rhNF- $\mathrm{kB}$ and DAPT indicated that Notch1 expression peaked on $7 \mathrm{~d}$ after treatment, suggesting that the change of Notch 1 signaling with intervention agent act as in the course of normal wound healing (Figure 2C).

Immunofluorescence double staining indicated that the number of BrdU/Hes1 double-positive cells were markedly increased in the rhNF- $\kappa \mathrm{B}$
A

B
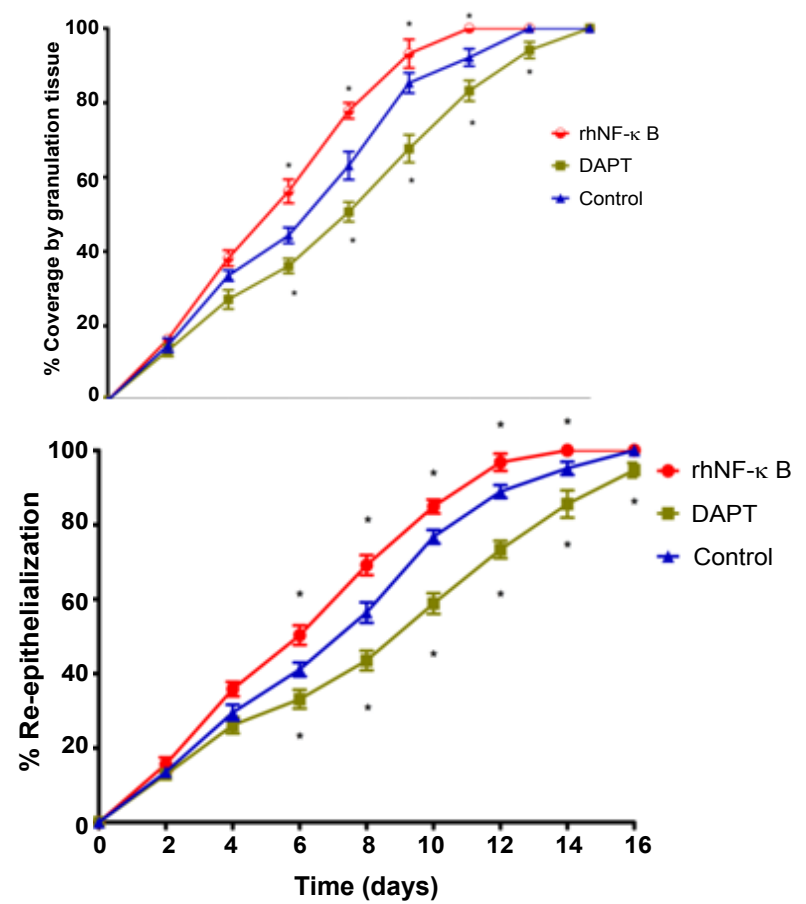
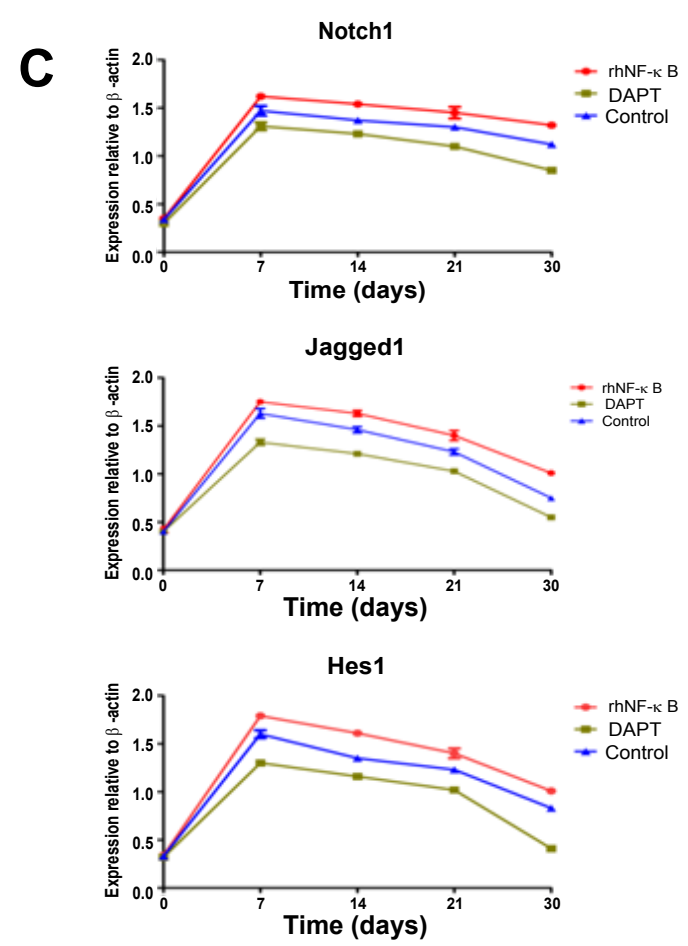

D



Figure 2: Wound healing is affected by Notch1 activator and inhibitor. (A) The rate of wound healing by measuring granulation and re-epithelialization. (B) rhNF-KB and DAPT changed wound healing rate compare with control rats. ${ }^{*} P<0.05$ (C) rhNF-KB up-regulated the expression of Notch1 signaling component, whereas DAPT administration inhibited Notch1 signaling pathway during wound healing. ${ }^{*} P<0.05$ (D) Immunofluorescence double staining indicated that the number of Brdu/Hes 1 double-positive cells were markedly increased in the rhNF-kB group, while DAPT group were significantly decreased compared with that of control group during wound repair. ${ }^{*} P<0.05$. 
group than in the control culture, while that of the DAPT group were significantly decreased compared with that of control group during wound repair (at least $\mathrm{p}<0.05$ ) (Figure 2D). These results implied that adjusting Notch1 signaling pathways would affect the expression of ESCs during wound healing.

\section{Stem cell characteristics of ESCs change when Notch1 signaling varies}

When ESCs were placed in a pellet culture system with JAG1, they displayed a rapid increase in colony-forming ability than in DAPTtreated and untreated cultures. MTT assay showed that activated Notch signaling by JAG1 significantly promoted ESCs proliferation (Figure $3 \mathrm{~A})$. Fluorescence-activated cell sorting (FACS) analysis showed that the percentage of K19 (an ESC surface marker)-positive cells in the JAGl group was significantly higher than that in the DAPT and control groups. However, the expression of K10 (a keratinocyte surface marker) was significantly lower in the JAGl group than that in the control group. By contrast, the expression of K10 in the DAPT group was significantly higher than that in the control group (Figure 3B). No morphological changes were observed in any of the groups after $48 \mathrm{~h}$ of culture. These observations indicate that JAG1 could maintain the "stemness" of ESCs by the activation of Notch1 and Wnt signaling.

The mRNA and protein expression levels of $\alpha$-SMA and collagen I in the DAPT group were significantly higher than those in the control group (Figures 3C and 3D). The a-SMA and collagen I expression levels were undetectable in the JAG1 group. In the experiments in which the Notch signaling pathway was inhibited by DAPT, ESCs exhibited a tendency to differentiate into keratinocytes and myofibroblasts.

\section{Notch signaling pathways are upregulated by jagged1 and downregulated by DAPT in ESCs}

Next, we tested whether Notch1 signaling of ESCs was regulated by Jagged 1 and DAPT. Upon the in vitro exposure of JAG1, ESCs exhibited significantly increased expression levels of NICD, Jagged1, and Hes1 (essential components of the Notch1 signaling pathway), as detected by immunohistochemistry, western blot, and quantitative reverse transcriptase-polymerase chain reaction (qRT-PCR) analysis. On the other hand, when ESCs were treated with DAPT, results have shown that DAPT treatment inhibited the expression of protein and mRNA in Notch1 signaling (Figure 4).
A



C



B

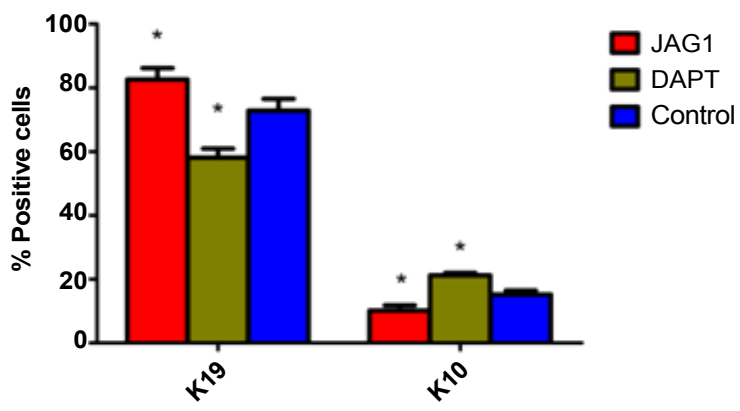

D

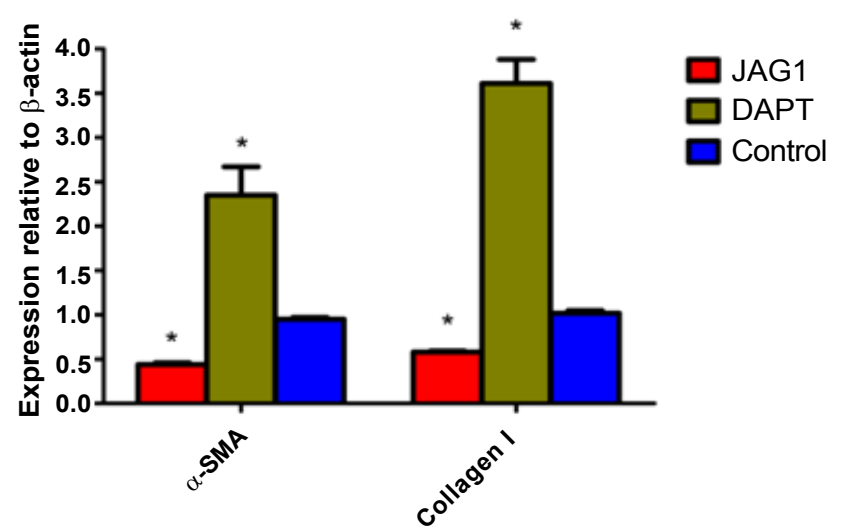

Figure 3: Stem Cell Characteristics of ESCs Change when Notch1 Signaling varies. (A) MTT assay showed that activated Notch signaling by JAG1 significantly promoted ESCs proliferation on $5 \mathrm{~d}$ and $7 \mathrm{~d}$. ${ }^{*} P<0.05$ (B) FACS analysis showed that the percentage of K19 positive cells in the JAGI group was significantly higher than that in the DAPT and control groups. By contrast, the expression of K10 in the DAPT group was significantly higher than that in the control group and JAG1 group. ${ }^{*} P<0.05$ (C) The mRNA expression levels of $\alpha$-SMA and collagen I in the DAPT group were significantly higher than those in the control group. ${ }^{*} P<0.05$ (D) Protein expression levels of $\alpha$-SMA and collagen I in the DAPT group were significantly higher than those in the control group. ${ }^{*} P<0.05$. 
A

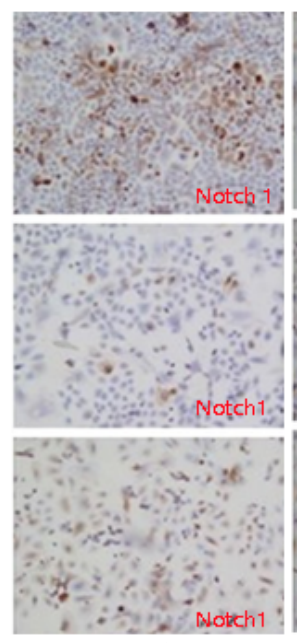

Notch1



DAPT

\section{B}
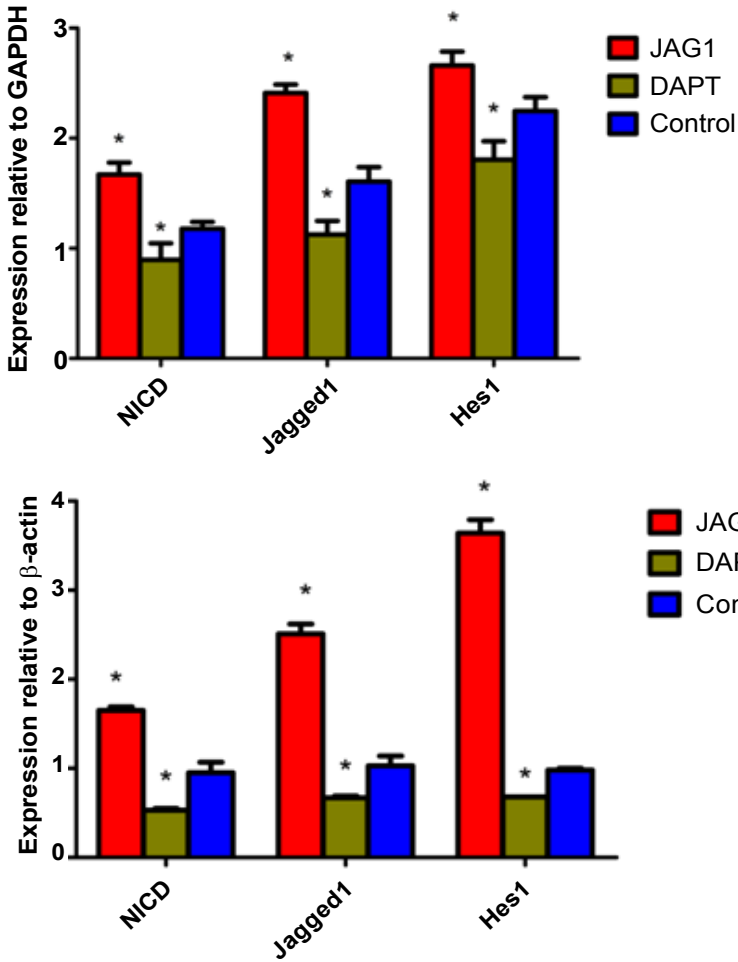

Figure 4: Notch Signaling Pathways are Upregulated by Jagged1 and Downregulated by DAPT in ESCs. (A) Immunohistochemistry for NICD, Jagged1, and Hes1 were activated by Jagged 1 and inhibited by DAPT. (B) qRT-PCR showed that mRNA of NICD, Jagged1, and Hes1 were activated by Jagged1 and inhibited by DAPT. ${ }^{*} P<0.05$ (C) Western blotting showed that protein of NICD, Jagged1, and Hes1 were activated by Jagged1 and inhibited by DAPT. ${ }^{*} P<0.05$.

\section{siRNA-mediated knockdown of jagged1 in ESCs affects wound healing}

Compared with the control mice treated with control siRNAtransfected ESCs, gross observation of wounds showed that mice treated with JAG1 siRNA-transfected ESCs exhibited significantly delayed wound healing (Figure 5A). Immunofluorescence staining of Collegan was performed to intuitively show that more regular and ordered collagen arrangement formed in control group (Figure 5B). Moreover, the positive expression of CD31 in the control group was significantly higher than that in the JAG1 siRNA group (Figure 5C). The hematoxylin and eosin (HE) staining results are presented a small amount of typical original skin appendages, such as hair follicles and sebaceous glands, were observed in the control siRNA group at the wound closure stage and new epidermis layer that formed after wound healing was significantly thicker in the control siRNA group than that in the JAG1 siRNA group, with the former having more cell layers, more epidermal ridges (Figure 5D).

\section{Discussion}

Wound healing is a complicated course involve in chemical signaling, cellular migration, extracellular deposition, proteolysis, and angiogenesis. It has been well known that ESCs contribute to homeostatic maintenance of the skin and wound repairing. During wound repairing, lost cells are eventually restored in a process initiated by proliferative basal cells consisting of ESCs. Recent studies have shown that there are three distinct ESCs niches, including bulge of the hair follicle (HF), the base of the sebaceous gland (SG), and the basal layer of the interfollicular epidermis (IFE) [15,17]. In response to wound repair, different reservoir ESCs would differentiate into cells of all epidermal lineages. Both HF and IFE niches participate in the course of wound re-epithelialization $[18,19]$, while SG play a vital role in the dremis remodel and hair follicle regeneration [20]. ESCs are regulated by a niche that includes the signaling pathways. Recent evidence has shed light on the interactions between ESCs and Notch signaling. Notch signaling is important in the determination of ESCs self-renewal and differentiation. Notch signaling was originally described as a critical pathway that inhibits cellular differentiation by inducing transcriptional repressors, such as Hes and Hes-related repressor protein family genes. The activation of Notch signaling is thought to increase the differentiation of ESCs into keratinocyte and IFE lineages [21]. In this study, we found that Notch1, the activated form of Notch1 (NICD), and its downstream target Hes1 were predominantly expressed in ESCs treated with JAG1, as well asJagged1. Simultaneously, our findings showed that activation of Notch1 signaling could promote ESC proliferation and maintaining their low differentiation and multi-directional differentiation potential. When the Notch signaling pathway was blocked ESCs proliferation was inhibited and ESCs had the tendency to differentiate into their terminal forms, such as epidermal cells and myofibroblasts.

Although the promoting effects of Notch1 signaling on wound healing have long been recognized, their underlying mechanism remains largely obscure. The role of ESCs in maintaining skin homeostatic and contributing to wound repair has been well acknowledged. In our finding, we were pleased to identify ESCs stained by BrdU are highly expressed in epidermis, which showed Hes1 high expression at the same time. Hes 1 is one of the major target genes in the Notch1 signaling 
Citation: Shu B, Yang RH, Shi Y, Xu YB, Liu J, et al. (2016) Notch1 Signaling Regulates Wound Healing via Changing the Characteristics of Epidermal Stem Cells. J Stem Cell Res Ther 6: 348. doi:10.4172/2157-7633.1000348
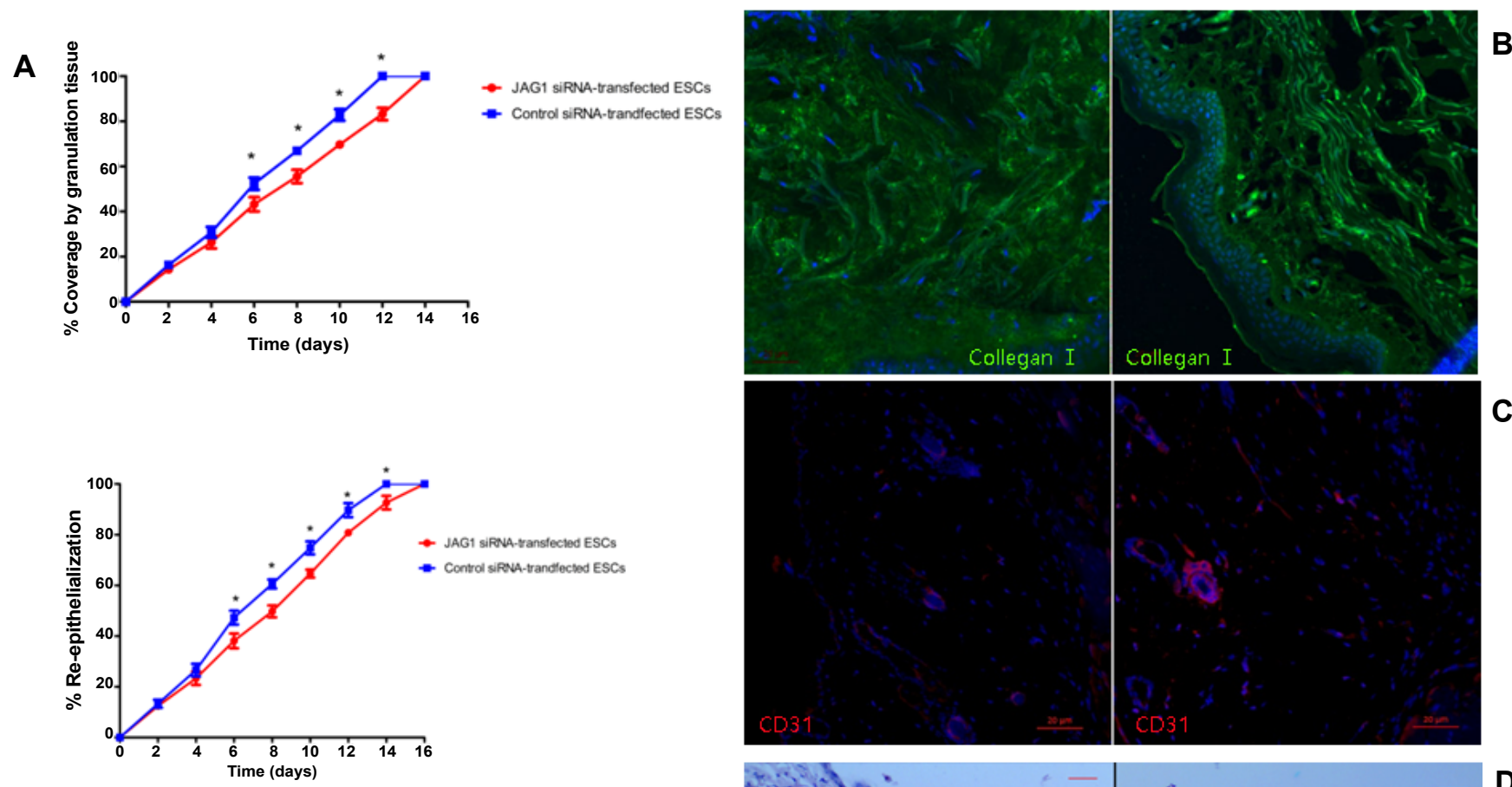

C

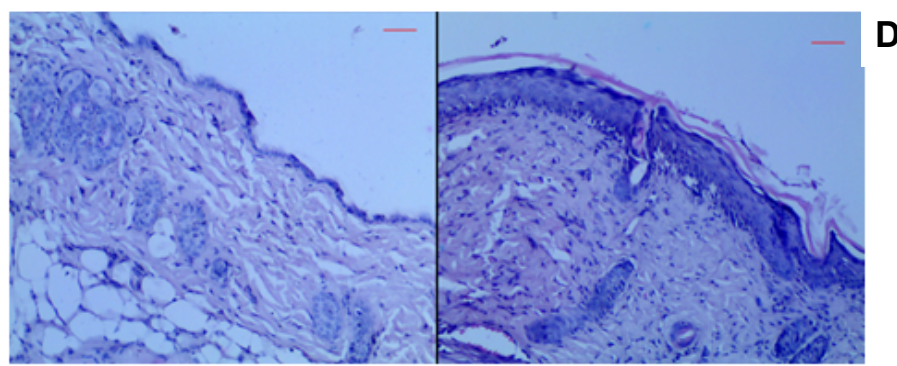

JAG1 siRNA-transfected ESCs

Control siRNA-transfected ESCs

Figure 5: siRNA-Mediated Knockdown of Jagged1 in ESCs affects wound healing. (A) Compared with the control mice treated with control siRNA-transfected ESCs, gross observation of wounds showed that mice treated with JAG1 siRNA-transfected ESCs exhibited significantly delayed wound healing. ${ }^{*} P<0.05$ (B) Immunofluorescence staining of Collegan I showed that more regular and ordered collagen arrangement formed in control group. (C) The positive expression of CD31 in the control group was significantly higher than that in the JAG1 siRNA group. (D) HE staining results are presented a small amount of typical original skin appendages, such as hair follicles and sebaceous glands, were observed in the control siRNA group at the wound closure stage and new epidermis layer that formed after wound healing was significantly thicker in the control siRNA group than that in the JAG1 siRNA group.

pathway, and it plays an essential role in maintaining the proliferation potential of stem cells [22]. Notch1 signaling plays an important role in maintaining the homeostasis of cutaneous epithelial cells. In normal adult skin, Notch1 mRNA transcript was detected in the basal layer of interfollicular epidermis which Jagged 1 appeared a similar distribution. In the course of wound healing, Notch signaling regulates inflammation, angiogenesis and epidermal differentiation, and that it is required for proper healing [23-25]. In response to wound, research has suggested Notch1 showed a gradation of increasing intensity from the wound centre to the edge was evident. The highest level of transcription of Notch1 signaling is in the basal cell layer of non-lesional skin and during the first step of re-epithelialisation, expression was weak or nonexistent [12]. Consistent with this, Notch1 gene expression in regenerated epidermis is lower in the middle than at the wound edges [26]. However, we observed Notch1 signaling components with low expression was localized to suprabasal cells in unwounded adult rat skin which was elevated after wound and remained high expression until 30d. A possible cause of these different results would be different experimental methods and possible posttranscriptional and/or post- translational control of protein levels leading to Notch1 protein accumulation in the wound site.

Our findings also suggest Notch1 expression varied in response to stimulation with either $\mathrm{rhNF}-\mathrm{\kappa B}$ or DAPT. In wound healing experiment, healing was delayed in Notch antisense transgenic mice and enhanced in Jagged- 1 treated mice [13]. Moreover, we observed that activation of Notch1 will result in enhanced wound closure, epidermis layer thicken, skin appendages regeneration and more regular and ordered collagen arrangement. These results also coincide with that blocking of Notch signaling decrease scar formation and inhibition of Notch signaling prevented experimental fibrosis [11,14,27] utilized Notch1 hemizygous mice discovered Notch1 increased vascularity in wounds and collagen deposition in wounds which wound tissue continued to be more organized than in wild-type littermates [14].

a-SMA (a myofibroblast marker) and extracellular matrix (ECM) components, such as collagen I and collagen III, are factors that are closely related to scar formation and contraction [28,29]. Similar to muscle cell contraction, myofibroblast contraction is dependent on a-SMA. After wound closure, the myofibroblasts decrease because 
of apoptosis and eventually disappear. Thus, scar formation and contraction occur easily when the apoptosis of collagen I and collagen III is inhibited. In this study, a-SMA expression in the JAG1 siRNA group peaked at $7 \mathrm{~d}$ after injury, which was delayed than that in the control group. The expression levels of proteins and mRNA in the JAG1 siRNA group at days $7,14,21$, and 30 were significantly higher than those in the control group. The long-term, high-level expression of a-SMA, especially after wound healing, may be one of the important reasons for the easy formation of scars in nude mice by Jagged1 knockdown. Although some studies found that $\alpha$-SMA can also lead to wound contraction [30], our results show that the healing speed in the control group was faster than that in the JAG1 siRNA group possibly because ESC proliferation was not inhibited in the control group. Therefore, early expression is possibly more conducive to wound contraction and repair. During normal wound healing and reconstruction, collagen I proteins are mainly replaced in the later stage by collagen III [31]. Thus, the ratio of collagen III/collagen I in normal skin tissues is higher than that in the scar tissue [32]. In the present study, the expression of collagen I in the JAG1 siRNA group was higher than that in the control group during wound healing. Collagen I did not show a tendency of being replaced by collagen III $30 \mathrm{~d}$ after the injury, which may be one of the important factors for scar formation.

These results implied that Notch1 may be involved in initiating ESCs to participate in the course of re-epithelization, extracellular matrix forming and skin appendages regeneration. Thus, this report provides new evidence for the potential function of Notch signaling in regulating ESCs differentiation and wound repair. Defining the function of Notch signaling pathway in ESCs allows the development of new therapeutic strategies for delayed healing and pathological scarring.

\section{Experimental Procedures}

All rodents were purchased from the Experimental Animal Center of Sun Yat-Sen University and kept under standard conditions according to the regulation of Ethical Committee of Medical Sciences Department. All animal procedures were approved under the guidelines of the ethics committee of the first affiliated hospital of Sun Yat-sen University.

\section{BrdU label-retaining and wound creation}

BrdU labet retaining cells were generated as previously reported [33]. Briefly, ten-day-old neonatal Sprague-Dawley rats were injected intraperitoneally with $50 \mu \mathrm{g} / \mathrm{g}$ body weight 5 -bromo-2'-deoxyuridine (BrdU, Sigma, USA) every $12 \mathrm{~h}$ for a total of four injections to label mitotic cells. All rats were randomly divided into three groups (rhNF- $\kappa \mathrm{B}$ group, DAPT group, and control group) $(\mathrm{N}=25)$. After 60 days, these rats were anesthetized with sodium pentobarbital. Two full-thickness wounds were made using a sterile biopsy punch with a diameter of $1.0 \mathrm{~cm}$ which were $1-1.5 \mathrm{~cm}$ off the midspinal line on each side of dorsal skin. rhNF- $\kappa B$ ( $1 \mathrm{mg} / \mathrm{l})$, DAPT $(20 \mathrm{mMol} / \mathrm{l})$ were topically and respectively applied to the wound under the occlusive dressing once daily until wound closure according to the requirement, allowing the solution to bathe the wound, whereas there was no any treatment in the control group. All animals were housed individually with daily dressing changes and wound site documentation.

\section{Wound analysis}

Digital photographs of the wounds were taken at days $0,2,4,6,8$, $10,12,14$ and 16. Time to wound closure was defined as the time at which the wound bed was completely re-epithelialized and filled with new tissue. Wound area was measured by tracing the wound margin and calculating for the area using an image analysis program. The percentage of wound closure was calculated as follows: (area of original wound - area of actual wound)/area of original wound $\times 100$. The inside edge of the splint exactly matched the edge of the wound. Thus, the splinted hole was used to represent the original wound size. The rats were sacrificed at postoperatively days $0,7,14,21$, and $30 \mathrm{~d}$. At which times skin samples, including the wound and $5 \mathrm{~mm}$ of the surrounding skin, were harvested using a $25 \mathrm{~mm}$ biopsy punch. For whole-mount skin samples, the entire wound and surrounding skin were placed on a plastic container (tissue culture dish) with the dermis side down, and the sample was photographed immediately and processed for Western Blot Analysis and histopathological evaluation.

\section{Immunohistochemistry and immunohistofluorescence}

Skin samples were fixed in $4 \%$ formalin solution, embedded in paraffin, and sectioned $(5 \mu \mathrm{m})$ for immunohistochemistry and immunohistofluorescence.

Immunohisochemistry staining was used to detected expression of Notch1. Paraffin sections were subjected to antigen retrieval using a pressure cooker, in sodium citrate ( $\mathrm{pH}$ 6.0), for 4 minutes. Endogenous peroxidase was blocked with $3 \%$ hydrogen peroxide $\left(\mathrm{H}_{2} \mathrm{O}_{2}\right)$ in PBS followed by nonspecific blocking with $2 \% \mathrm{PBS}+$ bovine serum albumin (BSA) for 15 minutes. The sections were incubated with the primary antibody overnight at $4^{\circ} \mathrm{C}$. After washing with PBS, slides treated with biotin-labeled secondary antibodies (1:300, R\&D, USA) were incubated at RT for $1 \mathrm{~h}$. The chromogenic reagent DAB was used to show the antibody conjugation. The intensity of the reaction observed on the slides was qualitatively analyzed.

Double-immunolabelling was used to detect Hes1 and BrdU in epidermis during wound healing. Formalin-fixed sections were deparaffinised in xylene and rehydrated in graded alcohols. Tissue sections were microwaved in $10 \mathrm{mM}$ sodium citrate $(\mathrm{pH}$ 6.0) for 3 minutes, incubated for another 15 minutes in the hot solution and rinsed in Automation Buffer (Biomedia, Foster City, CA). Sections were incubated in $2 \mathrm{M} \mathrm{HCl}$ at $37^{\circ} \mathrm{C}$, washed in borate buffer, and digested in $0.01 \%$ trypsin in $0.05 \mathrm{M}$ Tris for 3 minutes at $37^{\circ} \mathrm{C}$. After blocking in $10 \%$ goat serum for 20 minutes, sections were incubated for 1 hour at room temperature with mouse BrdU antisera (Becton Dickinson; 1:25) and Hes1 antisera (1:100) in 1\% bovine serum albumin.

\section{ESCs isolation and culture}

Pregnant Sprague-Dawley (SD) rats were obtained from the Experimental Animal Center of Sun Yat-Sen University and were kept under standard conditions according to the ethical committee of the Medical Sciences Department. In this study, we used fetal rats $(19 \mathrm{~d}$ to $21 \mathrm{~d}$ gestational age). After sacrificing the rats, the skin from the torso of each rat was taken, rinsed twice with D-Hanks buffer, and immersed in D-Hanks buffer containing $200 \mathrm{U} / \mathrm{mL}$ penicillin and $200 \mathrm{U} / \mathrm{mL}$ streptomycin (Hyclone, Cat. No. SH30010) for $30 \mathrm{~min}$. Under sterile conditions, the skin was washed thoroughly with PBS to remove the subcutaneous tissue, and trimmed into $0.5 \times 0.5 \mathrm{~cm}$ pieces. The skin was digested at $4^{\circ} \mathrm{C}$ overnight in digestion buffer containing $0.5 \%$ neutral protease (GIBCO Cat. No. 17105041). The following morning, the skin sample was incubated at $37^{\circ} \mathrm{C}$ for $30 \mathrm{~min}$. After peeling off the epidermis and cutting into the microskin, the skin sample was oscillated and digested with $0.25 \%$ trypsin (Hyclone, Cat. No. SH3008742.01) at $37^{\circ} \mathrm{C}$ for $15 \mathrm{~min}$ to prepare a single cell suspension. The digestion reaction was stopped by the addition of an equal volume of high-glucose Dulbecco's modified eagle's medium (DMEM) that contained 20\% 
fetal bovine serum (FBS) (Hyclone, Cat. No. SH30022.01B) relative to the volume of trypsin. The cells were filtered using a 200 mesh filter and centrifuged at $1,000 \mathrm{rpm}$ for $5 \mathrm{~min}$. After the removal of the supernatant, the cells were resuspended in high-glucose DMEM containing 20\% FBS and seeded at a cell density of $3 \times 10^{6} / \mathrm{mL}$ in a flask coated with type IV collagen (Sigma Cat. No. C8374). After $15 \mathrm{~min}$ at $37^{\circ} \mathrm{C}$, the cells were observed under an inverted phase contrast microscope, and some cells adhered to the flask. The suspended cells were collected, and $4 \mathrm{~mL}$ of high-glucose DMEM containing 20\% FBS (Hyclone, Cat. No. SH30022.01B) was added to the adherent cells. The cells were cultured at $5 \% \mathrm{CO} 2$ and saturated humidity at $37^{\circ} \mathrm{C}$. The culture medium was changed to K-SFM medium (GIBCO, Cat.No.17005042) after $24 \mathrm{~h}$, and the cells were passaged. Half of the medium was replaced every other day, and the complete medium was replaced every $2 \mathrm{~d}$ to $3 \mathrm{~d}$. When the culture reached $70 \%$ to $80 \%$ confluence, the cells were digested in $0.25 \%$ trypsin at $37^{\circ} \mathrm{C}$ with $5 \mathrm{~min}$ to $10 \mathrm{~min}$ of oscillation and passaged at a ratio of $1: 2$.

\section{Immunocytochemistry}

Cells were fixed with $4 \%$ paraformaldehyde for $30 \mathrm{~min}$, washed with PBS thrice for $5 \mathrm{~min}$, and incubated with $3 \%$ peroxide in a humidity box at room temperature for $10 \mathrm{~min}$. After three PBS washes for $5 \mathrm{~min}$ each, the cells were blocked with $10 \%$ normal goat serum at room temperature for $30 \mathrm{~min}$. Incubation with primary antibodies was performed at $4^{\circ} \mathrm{C}$ overnight. The primary antibodies used were rabbit anti-CK15 (BIOSS Cat. No. bs-1772R), rabbit anti-CK19 (BIOSS Cat. No. bs-1028R), and rabbit anti-P63 (BIOSS Cat. No. bs-0723R). Following $3 \times 5$ min washes with PBS, cells were incubated with the secondary antibody at room temperature for $30 \mathrm{~min}$, washed with PBS thrice for 5 min each, and incubated with DAB using ChemMate TM Dako Envision TM Detection Kit (Dako, GK500705). Staining was stopped by washing with PBS thrice. Cells were counterstained with hematoxylin. The superfluous stain was removed with water. The cells were dehydrated with $50 \%, 75 \%, 85 \%, 95 \%$, and $100 \%$ gradients of ethanol (once per step) and cleared with xylene twice for $10 \mathrm{~min}$ prior to mounting in neutral resin. An Olympus CX41 microscope was used to observe cells.

\section{Cell Proliferation assay}

Cells from the different groups were digested, dispersed by pipetting, and then counted. The cell concentration was adjusted to $1 \times 10^{5}$ cells $/ \mathrm{mL}$, and cells were distributed in a 96-well microplate (100 $\mu \mathrm{L} /$ well; i.e., $1 \times 10^{4}$ cells/well). After cell adhesion, the cells were collected at different time points $(0$ and $72 \mathrm{~h})$. MTS was added at a ratio of $1 / 10$ (i.e., $10 \mu \mathrm{L}$ of detection solution was added to $100 \mu \mathrm{L}$ of medium) according to the instructions in the CellTiter96 Aqueous One Solution Cell Proliferation Assay (MTS) (Promega, Cat. No. G3582). After $4 \mathrm{~h}$ of incubation, the MTS levels were read at OD 490 using a microplate reader (Thermo Fisher Scientific, Multiscan MK3).

\section{Marker detection by flow cytometry}

Cells were counted and the cell concentration was adjusted to $1 \times 10^{6}$ cells $/ 100 \mu \mathrm{L}$. A total of $100 \mu \mathrm{L}$ of cells was aliquoted into three tubes. Tube A was the blank control, tube B contained mouse IgG1 K isotype control PE, and tube $\mathrm{C}$ contained the fluorescein isothiocyanate (FITC)labeled hamster anti-rat CD29 (BD Pharmingen ${ }^{\mathrm{nx}}$, Cat. No. 555005). Antibodies were diluted (1:1) and incubated in a dark chamber at room temperature for $20 \mathrm{~min}$. The cells were washed with $500 \mu \mathrm{L}$ of PBS by centrifugation at $300 \mathrm{~g}$ for $10 \mathrm{~min}$. The cells were added with FITClabeled anti-mouse IgG (1:50; Abcam, Cat.No. ab6785), incubated for $20 \mathrm{~min}$, and washed once by centrifugation at $300 \mathrm{~g}$ for $10 \mathrm{~min}$. The cells were resuspended in $0.2 \mathrm{~mL}$ of PBS, and the reaction was analyzed by flow cytometry using a FACS Diva version 6.1 flow cytometer.

\section{Signaling pathway analysis}

ESCs were plated in six-well plates at a density of $10,000 \mathrm{cells} / \mathrm{cm}^{2}$ in K-SFM. To study the effects of Notch signaling crosstalk mediated by Jagged1, the medium was supplemented with either a Notch signaling activator, Jagged1/FC (1000 ng/mL; R\&D Systems), or a specific inhibitor, DAPT ( $16 \mu \mathrm{M}$; Sigma-Aldrich). Cells in the medium with PBS were used as the control group. The cells were harvested for RNA isolation after $5 \mathrm{~d}$ of culture. ESC proliferation and differentiation were investigated in a pellet culture system as described above. Samples were collected for RNA extraction and histological analysis after 3, 5, and $7 \mathrm{~d}$ of culture.

\section{MTT assay}

Cell growth was measured by MTT assay. MTT cell proliferation kits were purchased from BioVision Technologies (Exton, PA, USA). Cell proliferation was measured according to the manufacturer's protocol. The cells were portioned in a 96-well plate at 2,000 cells/ well to 5,000 cells/well. Cells were cultured in DMEM, either with or without FBS, depending on the individual experiments. Samples were assayed in triplicate and experiments were repeated thrice.

\section{siRNA gene silencing}

The siRNA targeting the rat form of Jagged1 (sc-61881), the non-targeting siRNA control, the transfection reagent, the siRNA transfection medium, and the siRNA dilution buffer were purchased from Santa Cruz Biotechnology. The experiments were performed according to the manufacturer's protocol.

\section{Wound healing model and ESC transplantation}

Twenty athymic nude mice (eight weeks old; female) were randomly divided into two groups, and two $10 \mathrm{~mm}$ full-thickness excisional skin wounds were created on each side of the midline after anesthesia. Each wound was treated with $1.0 \times 10^{6}$ cells (ESCs transfected with JAG1 siRNA or control siRNA) by implantation into the excisional wound, after which Tegaderm (3M, London, ON, Canada, http:// www. $3 \mathrm{~m} . c 0 \mathrm{~m})$ was placed over the wound. The animals were housed individually. We tested the adhesive on the skin of mice prior to this experiment and did not observe any skin irritation or allergic reaction in mice. Digital photographs of the wounds were taken at days $0,2,4$, $6,8,10,12,14$ and 16. Skin samples were harvested as described above at $16 \mathrm{~d}$.

\section{Histological and immunohistochemical studies}

Standard HE staining and dual-color immunofluorescence techniques were used throughout the study. For each antibody, staining was performed on at least three mice of each genotype, and the average staining intensity over the entire tissue area was scored. Representative images were obtained for each staining. Isotype-matched control antibodies (eBiosciences) were used as a negative control. For semiquantification, positive signals in at least five random high-power fields were visualized, counted, and expressed as the percentage of total DAPI-positive cells.

\section{Western blot analysis}

Cell lysates or rodents skin homogenates $(50 \mu \mathrm{g}$ to $100 \mu \mathrm{g}$ of total protein) were separated on a polyacrylamide-sodium dodecyl sulfate gel and electroblotted onto a nitrocellulose membrane (BioRad, Hercules, 
Citation: Shu B, Yang RH, Shi Y, Xu YB, Liu J, et al. (2016) Notch1 Signaling Regulates Wound Healing via Changing the Characteristics of Epidermal Stem Cells. J Stem Cell Res Ther 6: 348. doi:10.4172/2157-7633.1000348

Page 9 of 10

CA, USA). The proteins were incubated overnight with the antibodies, transferred to a PVDF membrane (Millipore, MA, USA), and detected for protein expression using an enhanced chemiluminescence (ECL; ECL Western Blot Substrate, Pierce, USA) system.

\section{RNA extraction and real-time PCR analysis}

RNA was extracted using a single-step method of TRIzol (Invitrogen). RNA concentration and purity were measured using a Nanodrop spectrophotometer, and cDNA was synthesized from $1 \mu \mathrm{g}$ of total RNA using RevertAid H minus first-strand cDNA synthesis kit (Fermentas) according to the manufacturer's instructions. Quantitative real-time PCR was performed with a 7500 Real-Time system using Fast SYBR Green Master Mix (Applied Biosystems) and the primers listed in manufacturer's instructions. After normalization to GAPDH mRNA, relative expression levels and fold induction of each target gene were calculated by comparative CT method [(1/2) formula, where $\triangle \mathrm{CT}$ is the difference between CT-target and CT-reference] using Microsoft Excel 2007.

\section{Statistical analysis}

All values are expressed as mean \pm SD. Student's paired t-test was performed for comparison of data of paired samples. Analysis of variance was used for multiple group comparisons, followed by Friedman's post-hoc test. A probability (p) value $<0.05$ was considered significant.

\section{Author Contributions}

B.S., R.H.Y. and Y.S. performed the experiments. Y.B.X., J.L. and P.W. contributed to the animal work. X.S.L. and S.H.Q. planned the experiments. J.L.X. planned the experiments, analyzed the data, and wrote the manuscript.

\section{Acknowledgments}

This work was supported by grants from the National Natural Science Foundation of China (81372062), the Guangdong Natural Science Foundation (2015A030313162) and Science and Technology Planning Project of Guangdong Province (2014A020212055 and 2013B022000053). This paper should be attributed to Department of Burns in the First Affiliated Hospital of Sun Yat-sen University. The authors have no conflicts of interest to disclose.

\section{References}

1. Trott A (1988) Mechanisms of surface soft tissue trauma. Ann Emerg Med 17: 1279-1283. [PubMed]

2. Barrientos S, Stojadinovic O, Golinko MS, Brem H, Canic MT (2008) Growth factors and cytokines in wound healing. Wound Repair Regen 16: 585-601. [PubMed]

3. Mascré G, Dekoninck S, Drogat B, Youssef KK, Broheé S, et al. (2012) Distinct contribution of stem and progenitor cells to epidermal maintenance. Nature 489: 257-262. [PubMed]

4. Ghazizadeh S, Taichman LB (2001) Multiple classes of stem cells in cutaneous epithelium: a lineage analysis of adult mouse skin. EMBO J 20: 1215-1222. [PubMed]

5. Kamstrup M, Faurschou A, Gniadecki R, Wulf HC (2008) Epidermal stem cells - role in normal, wounded and pathological psoriatic and cancer skin. Curr Stem Cell Res Ther 3: 146-150. [PubMed]

6. Peng LH, Mao ZY, Qi XT, Chen X, Li N, et al. (2013) Transplantation of bonemarrow-derived mesenchymal and epidermal stem cells contribute to wound healing with different regenerative features. Cell Tissue Res 352: 573-583. [PubMed]

7. Tumbar T, Guasch G, Greco V, Blanpain C, Lowry WE, et al. (2004) Defining the epithelial stem cell niche in skin. Science 303: 359-363. [PubMed]

8. Boehnke K, Hansen BF, Stark HJ, Boukamp P (2012) Stem cells of the human epidermis and their niche: composition and function in epidermal regeneration and carcinogenesis. Carcinogenesis 33: 1247-1258. [PubMed]
9. Rangarajan A, Talora C, Okuyama R, Nicolas M, Mammucari C, et al. (2001) Notch signaling is a direct determinant of keratinocyte growth arrest and entry into differentiation. EMBO J 20: 3427-3436. [PubMed]

10. Roy M, King TW (2013) Epidermal growth factor regulates NIKS keratinocyte proliferation through Notch signaling. J Surg Res 185: 6-11. [PubMed]

11. Syed F, Bayat A (2012) Notch signaling pathway in keloid disease: enhanced fibroblast activity in a Jagged-1 peptide-dependent manner in lesional vs. extralesional fibroblasts. Wound Repair Regen 20: 688-706. [PubMed]

12. Thélu J, Rossio P, Favier B (2002) Notch signalling is linked to epidermal cell differentiation level in basal cell carcinoma, psoriasis and wound healing. BMC Dermatol 2: 7 .

13. Chigurupati S, Arumugam TV, Son TG, Lathia JD, Jameel S, et al. (2007) Involvement of notch signaling in wound healing. PLoS One 2: e1167.

14. Outtz HH, Wu JK, Wang X, Kitajewski J (2010) Notch1 deficiency results in decreased inflammation during wound healing and regulates vascular endothelial growth factor receptor-1 and inflammatory cytokine expression in macrophages. J Immunol 185: 4363-4373. [PubMed]

15. Blanpain C, Fuchs E (2009) Epidermal homeostasis: a balancing act of stem cells in the skin. Nat Rev Mol Cell Biol 10: 207-217. [PubMed]

16. Watt FM, Estrach S, Ambler CA (2008) Epidermal Notch signalling: differentiation, cancer and adhesion. Curr Opin Cell Biol 20: 171-179. [PubMed]

17. Fuchs E (2008) Skin stem cells: rising to the surface. J Cell Biol 180: 273-284 [PubMed]

18. Morasso MI, Canic MT (2005) Epidermal stem cells: the cradle of epidermal determination, differentiation and wound healing. Biol Cell 97: 173-183. [PubMed]

19. Ito M, Cotsarelis $G$ (2008) Is the hair follicle necessary for normal wound healing? J Invest Dermatol 128: 1059-1061. [PubMed]

20. Zhao H, Qiao J, Zhang S, Zhang H, Lei X, et al. (2015) GPR39 marks specific cells within the sebaceous gland and contributes to skin wound healing. Sci Rep 5: 7913. [PubMed]

21. Ghadially R (2012) 25 years of epidermal stem cell research. J Invest Dermato 132: 797-810. [PubMed]

22. Chen BY, Wang X, Chen LW, Luo ZJ (2012) Molecular targeting regulation of proliferation and differentiation of the bone marrow-derived mesenchymal stem cells or mesenchymal stromal cells. Curr Drug Targets 13: 561-571. [PubMed]

23. Bielefeld KA, Nik SA, Alman BA (2013) Cutaneous wound healing: recruiting developmental pathways for regeneration. Cell Mol Life Sci 70: 2059-2081. [PubMed]

24. Li PN, Li H, Zhong LX, Sun Y, Yu LJ, et al. (2015) Molecular events underlying maggot extract promoted rat in vivo and human in vitro skin wound healing Wound Repair Regen 23: 65-73. [PubMed]

25. Xie F, Cai W, Liu Y, Li Y, Du B, et al. (2015) Vaccarin attenuates the human EA.hy926 endothelial cell oxidative stress injury through inhibition of Notch signaling. Int J Mol Med 35: 135-142. [PubMed]

26. Takazawa Y, Ogawa E, Saito R, Uchiyama R, Ikawa S, et al. (2015) Notch down-regulation in regenerated epidermis contributes to enhanced expression of interleukin-36 \pm and suppression of keratinocyte differentiation during wound healing. J Dermatol Sci 79: 10-19. [PubMed]

27. Dees C, Zerr P, Tomcik M, Beyer C, Horn A, et al. (2011). Inhibition of Notch signaling prevents experimental fibrosis and induces regression of established fibrosis. Arthritis Rheum 63: 1396-1404. [PubMed]

28. Sarrazy V, Billet $F$, Micallef $L$, Coulomb B, Desmoulière A (2011) Mechanisms of pathological scarring: role of myofibroblasts and current developments. Wound Repair Regen 19 Suppl 1: s10-s15. [PubMed]

29. Shin D, Minn KW (2004) The effect of myofibroblast on contracture of hypertrophic scar. Plast Reconstr Surg 113: 633-640. [PubMed]

30. Hinz B (2007) Formation and function of the myofibroblast during tissue repair. J Invest Dermatol 127: 526-537. [PubMed]

31. Gabbiani G, Le Lous M, Bailey AJ, Bazin S, Delaunay A (1976) Collagen and myofibroblasts of granulation tissue. A chemical, ultrastructural and immunologic study. Virchows Arch B Cell Pathol 21: 133-145. [PubMed]

32. Wang Q, Peng Z, Xiao S, Geng S, Yuan J, et al. (2007) RNAi-mediated 
Citation: Shu B, Yang RH, Shi Y, Xu YB, Liu J, et al. (2016) Notch1 Signaling Regulates Wound Healing via Changing the Characteristics of Epidermal Stem Cells. J Stem Cell Res Ther 6: 348. doi:10.4172/2157-7633.1000348

Page 10 of 10

inhibition of COL1A1 and COL3A1 in human skin fibroblasts. Exp Dermatol 16: 611-617. [PubMed]

33. Braun KM, Niemann C, Jensen UB, Sundberg JP, Vargas VS, et al. (2003)
Manipulation of stem cell proliferation and lineage commitment: visualisation of label-retaining cells in wholemounts of mouse epidermis. Development 130: 5241-5255. [PubMed] 